\title{
On perturbations of Dirac operators with variable magnetic field of constant direction
}

\author{
Serge Richard and Rafael Tiedra de Aldecoa \\ Département de Physique Théorique, Université de Genève, \\ 24, quai E. Ansermet, 1211 Genève 4, Switzerland \\ E-mails: serge.richard@physics.unige.ch and rafael.tiedra@physics.unige.ch
}

May 2004

\begin{abstract}
We carry out the spectral analysis of matrix valued perturbations of 3-dimensional Dirac operators with variable magnetic field of constant direction. Under suitable assumptions on the magnetic field and on the pertubations, we obtain a limiting absorption principle, we prove the absence of singular continuous spectrum in certain intervals and state properties of the point spectrum. Various situations, for example when the magnetic field is constant, periodic or diverging at infinity, are covered. The importance of an internal-type operator (a 2-dimensional Dirac operator) is also revealed in our study. The proofs rely on commutator methods.
\end{abstract}

\section{Introduction and main results}

We consider a relativistic spin- $\frac{1}{2}$ particle evolving in $\mathbb{R}^{3}$ in presence of a variable magnetic field of constant direction. By virtue of the Maxwell equations, we may assume with no loss of generality that the magnetic field has the form $\vec{B}\left(x_{1}, x_{2}, x_{3}\right)=\left(0,0, B\left(x_{1}, x_{2}\right)\right)$. So the unperturbed system is described, in the Hilbert space $L^{2}\left(\mathbb{R}^{3} ; \mathbb{C}^{4}\right)$, by the Dirac operator

$$
H_{0}:=\alpha_{1} \Pi_{1}+\alpha_{2} \Pi_{2}+\alpha_{3} P_{3}+\beta m,
$$

where $\beta \equiv \alpha_{0}, \alpha_{1}, \alpha_{2}, \alpha_{3}$ are the usual Dirac-Pauli matrices, $m$ is the strictly positive mass of the particle and $\Pi_{j}:=-i \partial_{j}-a_{j}$ are the generators of the magnetic translations with a vector potential $\vec{a}\left(x_{1}, x_{2}, x_{3}\right)=\left(a_{1}\left(x_{1}, x_{2}\right), a_{2}\left(x_{1}, x_{2}\right), 0\right)$ that satisfies $B=\partial_{1} a_{2}-\partial_{2} a_{1}$. Since $a_{3}=0$, we have written $P_{3}:=-i \partial_{3}$ instead of $\Pi_{3}$.

In this paper we study the stability of certain parts of the spectrum of $H_{0}$ under matrix valued perturbations $V$. More precisely, if $V$ satisfies some natural hypotheses, we shall prove the absence of singular continuous spectrum and the finiteness of the point spectrum of $H:=H_{0}+V$ in intervals of $\mathbb{R}$ corresponding to gaps in the symmetrized spectrum of the operator $H^{0}:=\sigma_{1} \Pi_{1}+\sigma_{2} \Pi_{2}+\sigma_{3} m$ in $\mathrm{L}^{2}\left(\mathbb{R}^{2} ; \mathbb{C}^{2}\right)$. The matrices $\sigma_{j}$ are the Pauli matrices and the symmetrized spectrum $\sigma_{\text {sym }}^{0}$ of $H^{0}$ is the 
union of the spectra of $H^{0}$ and $-H^{0}$. We stress that our analysis does not require any restriction on the behaviour of the magnetic field at infinity. Nevertheless, the pertinence of our work depends on a certain property of the internal-type operator $H^{0}$; namely, the size and the number of gaps in $\sigma_{\text {sym }}^{0}$. We refer to [2], [7], [10], [11] and [15] for various results on the spectrum of $H^{0}$, especially in the situations of physical interest, for example when $B$ is constant, periodic or diverges at infinity.

Technically, this work relies on commutator methods initiated by E. Mourre [13] and extensively developed in [1]. For brevity we shall constantly refer to the latter reference for notations and definitions. Our choice of a conjugate operator enables us to treat Dirac operators with general magnetic fields provided they point in a constant direction. On the other hand, as already put into evidence in [9], the use of a conjugate operator with a matrix structure has a few "rather awkward consequences" for long-range perturbations. We finally mention that this study is the counterpart for Dirac operators of [12], where only Schrödinger operators are considered. Unfortunately, the intrinsic structure of the Dirac equation prevents us from using the possible magnetic anisotropy to control the perturbations (see Remark 3.2 for details).

We give now a more precise description of our results. For simplicity we impose the continuity of the magnetic field and avoid perturbations with local singularities. Hence we assume that $B$ is a $C\left(\mathbb{R}^{2} ; \mathbb{R}\right)$-function and choose any vector potential $\vec{a}=\left(a_{1}, a_{2}, 0\right) \in C\left(\mathbb{R}^{2} ; \mathbb{R}^{3}\right)$, e.g. the one obtained by means of the transversal gauge [15]. The definitions below concern the admissible perturbations. In the long-range case, we restrict them to the scalar type in order not to impose unsatisfactory constraints. In the sequel, $\mathscr{B}_{\mathrm{h}}\left(\mathbb{C}^{4}\right)$ stands for the set of $4 \times 4$ hermitian matrices, and $\|\cdot\|$ denotes the norm of the Hilbert space $\mathcal{H}:=\mathrm{L}^{2}\left(\mathbb{R}^{3} ; \mathbb{C}^{4}\right)$ as well as the norm of $\mathscr{B}(\mathcal{H})$, the set of bounded linear operators on $\mathcal{H}$. $\mathbb{N}:=\{0,1,2, \ldots\}$ is the set of natural numbers. $\vartheta$ is an arbitrary $C^{\infty}([0, \infty))$-function such that $\vartheta=0$ near 0 and $\vartheta=1$ near infinity. $Q_{j}$ is the multiplication operator by the coordinate $x_{j}$ in $\mathcal{H}$, and the expression $\langle\cdot\rangle$ corresponds to $\sqrt{1+(\cdot)^{2}}$.

Definition 1.1. Let $V$ be a multiplication operator associated with an element of $\mathrm{L}^{\infty}\left(\mathbb{R}^{3} ; \mathscr{B}_{\mathrm{h}}\left(\mathbb{C}^{4}\right)\right)$.

(a) $V$ is small at infinity if $\lim _{r \rightarrow \infty}\left\|\vartheta\left(\frac{\langle Q\rangle}{r}\right) V\right\|=0$,

(b) $V$ is short-range if $\int_{1}^{\infty}\left\|\vartheta\left(\frac{\left\langle Q_{3}\right\rangle}{r}\right) V\right\| \mathrm{d} r<\infty$,

(c) Let $V_{L}$ be in $C^{1}\left(\mathbb{R}^{3} ; \mathbb{R}\right)$ with $x \mapsto\left\langle x_{3}\right\rangle\left(\partial_{j} V_{L}\right)(x)$ in $L^{\infty}\left(\mathbb{R}^{3} ; \mathbb{R}\right)$ for $j=1,2,3$, then $V:=V_{L}$ is long-range if

$$
\int_{1}^{\infty}\left\|\vartheta\left(\frac{\left\langle Q_{3}\right\rangle}{r}\right)\left\langle Q_{3}\right\rangle\left(\partial_{j} V\right)\right\| \frac{\mathrm{d} r}{r}<\infty \quad \text { for } \quad j=1,2,3
$$

Note that Definitions 1.1(b) and 1.1(c) differ from the standard ones: the decay rate is imposed only in the $x_{3}$ direction.

We are in a position to state our results. Let $\mathcal{D}\left(\left\langle Q_{3}\right\rangle\right)$ denote the domain of $\left\langle Q_{3}\right\rangle$ in $\mathcal{H}$, then the limiting absorption principle for $H$ is expressed in terms of the Banach space $\mathcal{G}:=\left(\mathcal{D}\left(\left\langle Q_{3}\right\rangle\right), \mathcal{H}\right)_{1 / 2,1}$ defined by real interpolation [1]. For convenience, we recall that $\mathcal{D}\left(\left\langle Q_{3}\right\rangle^{s}\right)$ is contained in $\mathcal{G}$ for each $s>1 / 2$. 
Theorem 1.2. Assume that $B$ belongs to $C\left(\mathbb{R}^{2} ; \mathbb{R}\right)$, and that $V$ belongs to $L^{\infty}\left(\mathbb{R}^{3} ; \mathscr{B}_{\mathrm{h}}\left(\mathbb{C}^{4}\right)\right)$, is small at infinity and can be written as the sum of a short-range and a long-range matrix valued function. Then

(a) The point spectrum of the operator $H$ in $\mathbb{R} \backslash \sigma_{\mathrm{sym}}^{0}$ is composed of eigenvalues of finite multiplicity and with no accumulation point in $\mathbb{R} \backslash \sigma_{\mathrm{sym}}^{0}$.

(b) The operator $H$ has no singular continuous spectrum in $\mathbb{R} \backslash \sigma_{\text {sym }}^{0}$.

(c) The limits $\lim _{\varepsilon \rightarrow+0}\left\langle\psi,(H-\lambda \mp i \varepsilon)^{-1} \psi\right\rangle$ exist for each $\psi \in \mathcal{G}$, uniformly in $\lambda$ on each compact subset of $\mathbb{R} \backslash\left\{\sigma_{\mathrm{sym}}^{0} \cup \sigma_{\mathrm{pp}}(H)\right\}$.

The above statements seem to be new for such a general magnetic field. In the special but important case of a nonzero constant magnetic field $B_{0}$, the admissible perturbations introduced in Definition 1.1 are more general than those allowed in [16]. We stress that in this situation $\sigma_{\text {sym }}^{0}$ is equal to $\left\{ \pm \sqrt{2 n B_{0}+m^{2}}: n \in \mathbb{N}\right\}$, which implies that there are plenty of gaps where our analysis gives results. On the other hand, if $B\left(x_{1}, x_{2}\right) \rightarrow 0$ as $\left|\left(x_{1}, x_{2}\right)\right| \rightarrow \infty$, our treatment gives no information since both $(-\infty,-m]$ and $[m, \infty)$ belong to $\sigma_{\mathrm{sym}}^{0}$. We finally mention the paper [3] for a related work on perturbations of magnetic Dirac operators.

\section{Mourre estimate for the operator $H_{0}$}

\subsection{Preliminaries}

Let us start by recalling some known results. The operator $H_{0}$ is essentially self-adjoint on $\mathscr{D}:=$ $C_{0}^{\infty}\left(\mathbb{R}^{3} ; \mathbb{C}^{4}\right)[5$. Thm. 2.1]. Its spectrum is symmetric with respect to 0 and does not contain the interval $(-m, m)$ [15] Cor. 5.14]. Thus the subset $H_{0} \mathscr{D}$ is dense in $\mathcal{H}$ since $\mathscr{D}$ is dense in $\mathcal{D}\left(H_{0}\right)$ (endowed with the graph topology) and $H_{0}$ is a homeomorphism from $\mathcal{D}\left(H_{0}\right)$ onto $\mathcal{H}$.

We now introduce a suitable representation of the Hilbert space $\mathcal{H}$. We consider the partial Fourier transformation

$$
\mathscr{F}: \mathscr{D} \rightarrow \int_{\mathbb{R}}^{\oplus} \mathcal{H}_{12} \mathrm{~d} \xi, \quad(\mathscr{F} \psi)(\xi):=\frac{1}{\sqrt{2 \pi}} \int_{\mathbb{R}} \mathrm{e}^{-i \xi x_{3}} \psi\left(\cdot, x_{3}\right) \mathrm{d} x_{3},
$$

where $\mathcal{H}_{12}:=\mathrm{L}^{2}\left(\mathbb{R}^{2} ; \mathbb{C}^{4}\right)$. This map extends uniquely to a unitary operator from $\mathcal{H}$ onto $\int_{\mathbb{R}}^{\oplus} \mathcal{H}_{12} \mathrm{~d} \xi$, which we denote by the same symbol $\mathscr{F}$. As a first application, one obtains the following direct integral decomposition of $H_{0}$ :

$$
\mathscr{F} H_{0} \mathscr{F}^{-1}=\int_{\mathbb{R}}^{\oplus} H_{0}(\xi) \mathrm{d} \xi,
$$

where $H_{0}(\xi)$ is a self-adjoint operator in $\mathcal{H}_{12}$ acting as $\alpha_{1} \Pi_{1}+\alpha_{2} \Pi_{2}+\alpha_{3} \xi+\beta m$ on $C_{0}^{\infty}\left(\mathbb{R}^{2} ; \mathbb{C}^{4}\right)$. In the following remark we draw the connection between the operator $H_{0}(\xi)$ and the operator $H^{0}$ introduced in Section 1 It reveals the importance of the internal-type operator $H^{0}$ and shows why its negative $-H_{0}$ also has to be taken into account.

Remark 2.1. The operator $H_{0}(0)$ acting on $C_{0}^{\infty}\left(\mathbb{R}^{2} ; \mathbb{C}^{4}\right)$ is unitarily equivalent to the direct sum operator $\left(\begin{array}{cc}m & \Pi_{-} \\ \Pi_{+} & -m\end{array}\right) \oplus\left(\begin{array}{cc}m & \Pi_{+} \\ \Pi_{-} & -m\end{array}\right)$ acting on $C_{0}^{\infty}\left(\mathbb{R}^{2} ; \mathbb{C}^{2}\right) \oplus C_{0}^{\infty}\left(\mathbb{R}^{2} ; \mathbb{C}^{2}\right)$, where $\Pi_{ \pm}:=\Pi_{1} \pm i \Pi_{2}$. 
Now, these two matrix operators act in $\mathrm{L}^{2}\left(\mathbb{R}^{2} ; \mathbb{C}^{2}\right)$ and are essentially self-adjoint on $C_{0}^{\infty}\left(\mathbb{R}^{2} ; \mathbb{C}^{2}\right)$ [5 Thm. 2.1]. However, the first one is nothing but $H^{0}$, while the second one is unitarily equivalent to $-H^{0}$ (this can be obtained by means of the abstract Foldy-Wouthuysen transformation [15] Thm. 5.13]). Therefore $H_{0}(0)$ is essentially self-adjoint on $C_{0}^{\infty}\left(\mathbb{R}^{2} ; \mathbb{C}^{4}\right)$ and

$$
\sigma\left[H_{0}(0)\right]=\sigma\left(H^{0}\right) \cup \sigma\left(-H^{0}\right) \equiv \sigma_{\text {sym }}^{0} .
$$

Moreover, there exists a relation between $\sigma\left[H_{0}(\xi)\right]$ and $\sigma_{\mathrm{sym}}^{0}$. Indeed, for $\xi \in \mathbb{R}$ fixed, one can show that $H_{0}(\xi)^{2}=H_{0}(0)^{2}+\xi^{2}$ on $\mathcal{D}\left(H_{0}(\xi)^{2}\right)=\mathcal{D}\left(H_{0}(0)^{2}\right)$, so that

$$
\sigma\left[H_{0}(\xi)^{2}\right]=\sigma\left[H_{0}(0)^{2}+\xi^{2}\right]=\left(\sigma\left[H_{0}(0)\right]\right)^{2}+\xi^{2}=\left(\sigma_{\text {sym }}^{0}\right)^{2}+\xi^{2},
$$

where the spectral theorem has been used for the second equality. Since the spectrum of $H_{0}(\xi)$ is symmetric with respect to 0 [15] Cor. 5.14], it follows that

$$
\sigma\left[H_{0}(\xi)\right]=-\sqrt{\left(\sigma_{\text {sym }}^{0}\right)^{2}+\xi^{2}} \cup \sqrt{\left(\sigma_{\text {sym }}^{0}\right)^{2}+\xi^{2}} .
$$

Define $\mu_{0}:=\inf \left|\sigma_{\mathrm{sym}}^{0}\right|$ (which is bigger or equal to $m$ because $H^{0}$ has no spectrum in $(-m, m)[15$ Cor. 5.14]). Then from the direct integral decomposition of $H_{0}$, one readily gets

$$
\sigma\left(H_{0}\right)=\left(-\infty,-\mu_{0}\right] \cup\left[\mu_{0},+\infty\right)
$$

We conclude the section by giving two technical lemmas in relation with the operator $H_{0}^{-1}$. Proofs can be found in an appendix.

Lemma 2.2. (a) For each $n \in \mathbb{N}, H_{0}^{-n} \mathscr{D}$ belongs to $\mathcal{D}\left(Q_{3}\right)$,

(b) $P_{3} H_{0}^{-1}$ is a bounded self-adjoint operator equal to $H_{0}^{-1} P_{3}$ on $\mathcal{D}\left(P_{3}\right)$. In particular, $H_{0}^{-1} \mathcal{H}$ belongs to $\mathcal{D}\left(P_{3}\right)$.

One may observe that, given a $C^{1}(\mathbb{R} ; \mathbb{C})$-function $f$ with $f^{\prime}$ bounded, the operator $f\left(Q_{3}\right)$ is welldefined on $\mathcal{D}\left(Q_{3}\right)$. Thus $f\left(Q_{3}\right) H_{0}^{-n} \mathscr{D}$ is a subset of $\mathcal{H}$ for each $n \in \mathbb{N}$. The preceding lemma and the following simple statement are constantly used in the sequel.

Lemma 2.3. Let $f$ be in $C^{1}(\mathbb{R} ; \mathbb{C})$ with $f^{\prime}$ bounded, and $n \in \mathbb{N}$. Then

(a) $i H_{0}^{-1} f\left(Q_{3}\right)-i f\left(Q_{3}\right) H_{0}^{-1}$ is equal to $-H_{0}^{-1} \alpha_{3} f^{\prime}\left(Q_{3}\right) H_{0}^{-1}$ on $H_{0}^{-n} \mathscr{D}$,

(b) $P_{3} H_{0}^{-1} f\left(Q_{3}\right)-f\left(Q_{3}\right) P_{3} H_{0}^{-1}$ is equal to $i\left(P_{3} H_{0}^{-1} \alpha_{3}-1\right) f^{\prime}\left(Q_{3}\right) H_{0}^{-1}$ on $\mathscr{D}$.

Both right terms belong to $\mathscr{B}(\mathcal{H})$. For shortness we shall denote them by $\left[i H_{0}^{-1}, f\left(Q_{3}\right)\right]$ and $\left[P_{3} H_{0}^{-1}, f\left(Q_{3}\right)\right]$ respectively.

\subsection{The conjugate operator}

The aim of the present section is to define an appropriate operator conjugate to $H_{0}$. To begin with, one observes that $Q_{3} P_{3} H_{0}^{-1} \mathscr{D} \subset \mathcal{H}$ as a consequence of Lemma 2.2 In particular, the formal expression

$$
A:=\frac{1}{2}\left(H_{0}^{-1} P_{3} Q_{3}+Q_{3} P_{3} H_{0}^{-1}\right)
$$

leads to a well-defined symmetric operator on $\mathscr{D}$. 
Proposition 2.4. The operator $A$ is essentially self-adjoint on $\mathscr{D}$ and its closure is essentially selfadjoint on any core for $\left\langle Q_{3}\right\rangle$.

Proof. The claim is a consequence of Nelson's criterion of essential self-adjointness [14 Thm. X.37] applied to the triple $\left\{\left\langle Q_{3}\right\rangle, A, \mathscr{D}\right\}$. Let us simply verify the two hypotheses of that theorem. By using Lemmas 2.2 and 2.3 one first obtains that for all $\psi \in \mathscr{D}$ :

$$
\|A \psi\|=\left\|\left(P_{3} H_{0}^{-1} Q_{3}-\frac{1}{2}\left[P_{3} H_{0}^{-1}, Q_{3}\right]\right) \psi\right\| \leq \mathrm{C}\left\|\left\langle Q_{3}\right\rangle \psi\right\|
$$

for some constant $\mathrm{C}>0$ independent of $\psi$. Then, for all $\psi \in \mathscr{D}$ one has:

$$
\begin{aligned}
\left\langle A \psi,\left\langle Q_{3}\right\rangle \psi\right\rangle-\left\langle\left\langle Q_{3}\right\rangle \psi, A \psi\right\rangle & =i \operatorname{Im}\left\langle Q_{3} \psi,\left[P_{3} H_{0}^{-1},\left\langle Q_{3}\right\rangle\right] \psi\right\rangle \\
& =i \operatorname{Re}\left\langle\left(\alpha_{3} P_{3} H_{0}^{-1}-1\right) Q_{3} \psi, Q_{3}\left\langle Q_{3}\right\rangle^{-1} H_{0}^{-1} \psi\right\rangle
\end{aligned}
$$

A few more commutator calculations, using again Lemma 2.3 with $f\left(Q_{3}\right)=\left\langle Q_{3}\right\rangle^{1 / 2}$, lead to the following result: for all $\psi \in \mathscr{D}$, there exists a constant $\mathrm{D}>0$ independent of $\psi$ such that

$$
\left|\left\langle A \psi,\left\langle Q_{3}\right\rangle \psi\right\rangle-\left\langle\left\langle Q_{3}\right\rangle \psi, A \psi\right\rangle\right| \leq \mathrm{D}\left\|\left\langle Q_{3}\right\rangle^{\frac{1}{2}} \psi\right\|^{2} .
$$

As far as we know, the matrix conjugate operator 2.4 has never been employed before for the study of magnetic Dirac operators.

\subsection{Strict Mourre estimate for $H_{0}$}

We now gather some results on the regularity of $H_{0}$ with respect to $A$. We recall that $\mathcal{D}\left(H_{0}\right)^{*}$ is the adjoint space of $\mathcal{D}\left(H_{0}\right)$ and that one has the continuous dense embeddings $\mathcal{D}\left(H_{0}\right) \hookrightarrow \mathcal{H} \hookrightarrow \mathcal{D}\left(H_{0}\right)^{*}$, where $\mathcal{H}$ is identified with its adjoint through the Riesz isomorphism.

Proposition 2.5. (a) The quadratic form $\mathcal{D}(A) \ni \psi \mapsto\left\langle H_{0}^{-1} \psi, i A \psi\right\rangle-\left\langle A \psi, i H_{0}^{-1} \psi\right\rangle$ extends uniquely to the bounded form defined by the operator $-H_{0}^{-1}\left(P_{3} H_{0}^{-1}\right)^{2} H_{0}^{-1} \in \mathscr{B}(\mathcal{H})$.

(b) The group $\left\{e^{i t A}\right\}_{t \in \mathbb{R}}$ leaves $\mathcal{D}\left(H_{0}\right)$ invariant.

(c) The quadratic form

$$
\mathcal{D}(A) \ni \psi \mapsto\left\langle H_{0}^{-1}\left(P_{3} H_{0}^{-1}\right)^{2} H_{0}^{-1} \psi, i A \psi\right\rangle-\left\langle A \psi, i H_{0}^{-1}\left(P_{3} H_{0}^{-1}\right)^{2} H_{0}^{-1} \psi\right\rangle,
$$

extends uniquely to a bounded form on $\mathcal{H}$.

In the framework of [1], the statements of (a) and (c) mean that $H_{0}$ is of class $C^{1}(A)$ and $C^{2}(A)$ respectively.

Proof. (a) For any $\psi \in \mathscr{D}$, one gets

$$
\begin{aligned}
2\left(\left\langle H_{0}^{-1} \psi, i A \psi\right\rangle-\left\langle A \psi, i H_{0}^{-1} \psi\right\rangle\right) & =\left\langle\left[i H_{0}^{-1}, Q_{3}\right] \psi, P_{3} H_{0}^{-1} \psi\right\rangle+\left\langle P_{3} H_{0}^{-1} \psi,\left[i H_{0}^{-1}, Q_{3}\right] \psi\right\rangle \\
& =-\left\langle H_{0}^{-1} \psi,\left(\alpha_{3} P_{3} H_{0}^{-1}+H_{0}^{-1} \alpha_{3} P_{3}\right) H_{0}^{-1} \psi\right\rangle
\end{aligned}
$$


where we have used Lemmas 2.2 and 2.3. Furthermore, one has

$$
H_{0}^{-1} \alpha_{3}=-\alpha_{3} H_{0}^{-1}+2 H_{0}^{-1} P_{3} H_{0}^{-1}
$$

as an operator identity in $\mathscr{B}(\mathcal{H})$. When inserting 2.7 into 2.6 , one obtains the equality

$$
\left\langle H_{0}^{-1} \psi, i A \psi\right\rangle-\left\langle A \psi, i H_{0}^{-1} \psi\right\rangle=-\left\langle\psi, H_{0}^{-1}\left(P_{3} H_{0}^{-1}\right)^{2} H_{0}^{-1} \psi\right\rangle .
$$

Since $\mathscr{D}$ is a core for $A$, the statement is obtained by density. We shall write $\left[i H_{0}^{-1}, A\right]$ for the bounded extension of the quadratic form $\mathcal{D}(A) \ni \psi \mapsto\left\langle H_{0}^{-1} \psi, i A \psi\right\rangle-\left\langle A \psi, i H_{0}^{-1} \psi\right\rangle$.

(b) Since $\mathcal{D}\left(H_{0}\right)$ is not explicitly known, one has to invoke an abstract result in order to show the invariance. Let $\left[i H_{0}, A\right]$ be the operator in $\mathscr{B}\left(\mathcal{D}\left(H_{0}\right), \mathcal{D}\left(H_{0}\right)^{*}\right)$ associated with the unique extension to $\mathcal{D}\left(H_{0}\right)$ of the quadratic form $\psi \mapsto\left\langle H_{0} \psi, i A \psi\right\rangle-\left\langle A \psi, i H_{0} \psi\right\rangle$ defined for all $\psi \in \mathcal{D}\left(H_{0}\right) \cap \mathcal{D}(A)$. Then $\mathcal{D}\left(H_{0}\right)$ is invariant under $\left\{e^{i t A}\right\}_{t \in \mathbb{R}}$ if $H_{0}$ is of class $C^{1}(A)$ and if $\left[i H_{0}, A\right] \mathcal{D}\left(H_{0}\right) \subset \mathcal{H}[8$, Lemma 2]. From equation (2.8) and [1 Eq. 6.2.24], one obtains the following equalities valid in form sense on $\mathcal{H}$ :

$$
-H_{0}^{-1}\left(P_{3} H_{0}^{-1}\right)^{2} H_{0}^{-1}=\left[i H_{0}^{-1}, A\right]=-H_{0}^{-1}\left[i H_{0}, A\right] H_{0}^{-1} .
$$

Thus $\left[i H_{0}, A\right]$ and $\left(P_{3} H_{0}^{-1}\right)^{2}$ are equal as operators in $\mathscr{B}\left(\mathcal{D}\left(H_{0}\right), \mathcal{D}\left(H_{0}\right)^{*}\right)$. But since the latter belongs to $\mathscr{B}(\mathcal{H}),[i H, A] \mathcal{D}\left(H_{0}\right)$ is included in $\mathcal{H}$.

(c) The boundedness on $\mathscr{D}$ of the quadratic form (2.5) follows by inserting (2.4) into the r.h.s. term of 2.5 and by applying repeatedly Lemma 2.3 with $f\left(Q_{3}\right)=Q_{3}$. Then one concludes by using the density of $\mathscr{D}$ in $\mathcal{D}(A)$.

From now on we shall simply denote the closure in $\mathcal{H}$ of $\left[i H_{0}, A\right]$ by $T=\left(P_{3} H_{0}^{-1}\right)^{2} \in \mathscr{B}(\mathcal{H})$. One interest of this operator is that $\mathscr{F} T \mathscr{F}^{-1}$ is boundedly decomposable [6 Prop. 3.6], more precisely:

$$
\mathscr{F} T \mathscr{F}^{-1}=\int_{\mathbb{R}}^{\oplus} T(\xi) \mathrm{d} \xi \quad \text { with } \quad T(\xi)=\xi^{2} H_{0}(\xi)^{-2} \in \mathscr{B}\left(\mathcal{H}_{12}\right) .
$$

In the following definition, we introduce two functions giving the optimal value to a Mourretype inequality. Remark that slight modifications have been done with regard to the usual definition [1, Sec. 7.2.1].

Definition 2.6. Let $H$ be a self-adjoint operator in a Hilbert space $\mathcal{H}$ and assume that $S$ is a symmetric operator in $\mathscr{B}\left(\mathcal{D}(H), \mathcal{D}(H)^{*}\right)$. Let $E^{H}(\lambda ; \varepsilon):=E^{H}((\lambda-\varepsilon, \lambda+\varepsilon))$ be the spectral projection of $H$ for the interval $(\lambda-\varepsilon, \lambda+\varepsilon)$. Then, for all $\lambda \in \mathbb{R}$ and $\varepsilon>0$, we set

$$
\begin{aligned}
\varrho_{H}^{S}(\lambda ; \varepsilon) & :=\sup \left\{a \in \mathbb{R}: E^{H}(\lambda ; \varepsilon) S E^{H}(\lambda ; \varepsilon) \geq a E^{H}(\lambda ; \varepsilon)\right\}, \\
\varrho_{H}^{S}(\lambda) & :=\sup _{\varepsilon>0} \varrho_{H}^{S}(\lambda ; \varepsilon) .
\end{aligned}
$$

Let us make three observations: the inequality $\varrho_{H}^{S}\left(\lambda ; \varepsilon^{\prime}\right) \leq \varrho_{H}^{S}(\lambda ; \varepsilon)$ holds whenever $\varepsilon^{\prime} \geq \varepsilon$, $\varrho_{H}^{S}(\lambda)=+\infty$ if $\lambda$ does not belong to the spectrum of $H$, and $\varrho_{H}^{S}(\lambda) \geq 0$ for all $\lambda \in \mathbb{R}$ if $S \geq 0$. We also mention that in the case of two self-adjoint operators $H$ and $A$ in $\mathcal{H}$, with $H$ of class $C^{1}(A)$ and $S:=[i H, A]$, the function $\varrho_{H}^{S}(\cdot)$ is equal to the function $\varrho_{H}^{A}(\cdot)$ defined in [1] Eq. 7.2.4]. 
Taking advantage of the direct integral decomposition of $H_{0}$ and $T$, one obtains for all $\lambda \in \mathbb{R}$ and $\varepsilon>0$ :

$$
\varrho_{H_{0}}^{T}(\lambda ; \varepsilon)=\underset{\xi \in \mathbb{R}}{\operatorname{essinf}} \varrho_{H_{0}(\xi)}^{T(\xi)}(\lambda ; \varepsilon) .
$$

Now we can deduce a lower bound for $\varrho_{H_{0}}^{T}(\cdot)$.

Proposition 2.7. One has

$$
\varrho_{H_{0}}^{T}(\lambda) \geq \inf \left\{\frac{\lambda^{2}-\mu^{2}}{\lambda^{2}}: \mu \in \sigma_{\text {sym }}^{0} \cap[0,|\lambda|]\right\}
$$

with the convention that the infimum over an empty set is $+\infty$.

Proof. We first consider the case $\lambda \geq 0$.

(i) Recall from (2.3 that $\mu_{0} \equiv \inf \left|\sigma_{\text {sym }}^{0}\right|=\inf \left\{\sigma\left(H_{0}\right) \cap[0,+\infty)\right\}$. Thus, for $\lambda \in\left[0, \mu_{0}\right)$ the 1.h.s. term of (2.10) is equal to $+\infty$, since $\lambda$ does not belong to the spectrum of $H_{0}$. Hence 2.10) is satisfied on $\left[0, \mu_{0}\right)$.

(ii) If $\lambda \in \sigma_{\text {sym }}^{0}$, then the r.h.s. term of 2.10 is equal to 0 . However, since $T$ is positive, $\varrho_{H_{0}}^{T}(\lambda) \geq 0$. Hence the relation $(2.10)$ is again satisfied.

(iii) Let $0<\varepsilon<\mu_{0}<\lambda$. Direct computations using the explicit form of $T(\xi)$ and the spectral theorem for the operator $H_{0}(\xi)$ show that for $\xi$ fixed, one has

$$
\varrho_{H_{0}(\xi)}^{T(\xi)}(\lambda ; \varepsilon)=\inf \left\{\frac{\xi^{2}}{\rho^{2}}: \rho \in(\lambda-\varepsilon, \lambda+\varepsilon) \cap \sigma\left[H_{0}(\xi)\right]\right\} \geq \frac{\xi^{2}}{(\lambda+\varepsilon)^{2}} .
$$

On the other hand one has $\varrho_{H_{0}(\xi)}^{T(\xi)}(\lambda ; \varepsilon)=+\infty$ if $(\lambda-\varepsilon, \lambda+\varepsilon) \cap \sigma\left[H_{0}(\xi)\right]=\varnothing$, and a fortiori

$$
\varrho_{H_{0}(\xi)}^{T(\xi)}(\lambda ; \varepsilon)=+\infty \quad \text { if } \quad\left((\lambda-\varepsilon)^{2},(\lambda+\varepsilon)^{2}\right) \cap \sigma\left[H_{0}(\xi)^{2}\right]=\varnothing .
$$

Thus, by taking into account equation (2.9), 2.11, the previous observation and relation 2.2], one obtains that

$$
\varrho_{H_{0}}^{T}(\lambda ; \varepsilon) \geq \operatorname{essinf}\left\{\frac{\xi^{2}}{(\lambda+\varepsilon)^{2}}: \xi^{2} \in\left((\lambda-\varepsilon)^{2},(\lambda+\varepsilon)^{2}\right)-\left(\sigma_{\text {sym }}^{0}\right)^{2}\right\} .
$$

Suppose now that $\lambda \notin \sigma_{\text {sym }}^{0}$, define $\mu:=\sup \left\{\sigma_{\text {sym }}^{0} \cap[0, \lambda]\right\}$ and choose $\varepsilon>0$ such that $\mu<\lambda-\varepsilon$. Then the inequality $(2.12)$ implies that

$$
\varrho_{H_{0}}^{T}(\lambda ; \varepsilon) \geq \frac{(\lambda-\varepsilon)^{2}-\mu^{2}}{(\lambda+\varepsilon)^{2}} .
$$

Hence the relation (2.10) follows from the above formula when $\varepsilon \rightarrow 0$.

For $\lambda<0$, similar arguments lead to the inequality

$$
\varrho_{H_{0}}^{T}(\lambda) \geq \inf \left\{\frac{\lambda^{2}-\mu^{2}}{\lambda^{2}}: \mu \in \sigma_{\text {sym }}^{0} \cap[\lambda, 0]\right\} .
$$

The claim is then a direct consequence of the symmetry of $\sigma_{\text {sym }}^{0}$ with respect to 0 .

The above proposition implies that we have a strict Mourre estimate, i.e. $\varrho_{H_{0}}^{T}(\cdot)>0$, on $\mathbb{R} \backslash \sigma_{\text {sym }}^{0}$. Moreover it is not difficult to prove that $\varrho_{H_{0}}^{T}(\lambda)=0$ whenever $\lambda \in \sigma_{\text {sym }}^{0}$. It follows that the conjugate operator $A$ does not allow to get spectral informations on $H_{0}$ in the subset $\sigma_{\text {sym }}^{0}$. 


\section{Mourre estimate for the perturbed Hamiltonian}

In the sequel, we consider the self-adjoint operator $H:=H_{0}+V$ with a potential $V$ that belongs to $\mathrm{L}^{\infty}\left(\mathbb{R}^{3} ; \mathscr{B}_{\mathrm{h}}\left(\mathbb{C}^{4}\right)\right)$. The domain of $H$ is equal to the domain $\mathcal{D}\left(H_{0}\right)$ of $H_{0}$. We first give a result on the difference of the resolvents $(H-z)^{-1}-\left(H_{0}-z\right)^{-1}$ and, as a corollary, we obtain the localization of the essential spectrum of $H$.

Proposition 3.1. Assume that $V$ is small at infinity. Then for all $z \in \mathbb{C} \backslash\left(\sigma(H) \cup \sigma\left(H_{0}\right)\right)$ the difference $(H-z)^{-1}-\left(H_{0}-z\right)^{-1}$ is a compact operator. It follows in particular that $\sigma_{\mathrm{ess}}(H)=\sigma_{\mathrm{ess}}\left(H_{0}\right)$.

Proof. Since $V$ is bounded and small at infinity, it is enough to check that $H_{0}$ is locally compact [15, Sec. 4.3.4]. However, the continuity of $\vec{a}$ implies that $\mathcal{D}\left(H_{0}\right) \subset \mathcal{H}_{\mathrm{loc}}^{1 / 2}$ [4] Thm. 1.3]. Hence the statement follows by usual arguments.

Remark 3.2. In the study of an analogous problem for Schrödinger operators [12], the authors prove a result similar to Proposition 3.1 without assuming that the perturbation is small at infinity (it only has to be small with respect to $B$ in a suitable sense). Their proof mainly relies on the structural inequalities $H_{\text {Sch }}:=\Pi_{1}^{2}+\Pi_{2}^{2}+P_{3}^{2} \geq \pm B$. In the Dirac case, the counterpart of these turn out to be

$$
H_{0}^{2} \geq 2 B \cdot \operatorname{diag}(0,1,0,1) \quad \text { and } \quad H_{0}^{2} \geq-2 B \cdot \operatorname{diag}(1,0,1,0),
$$

where $\operatorname{diag}(\cdots)$ stands for a diagonal matrix. If we assume that the magnetic field is bounded from below, the first inequality enables us to treat pertubations of the type $\operatorname{diag}\left(V_{1}, V_{2}, V_{3}, V_{4}\right)$ with $V_{2}, V_{4}$ small with respect to the magnetic field and $V_{1}, V_{3}$ small at infinity in the original sense. If the magnetic field is bounded from above, the second inequality has to be used and the role of $V_{2}, V_{4}$ and $V_{1}, V_{3}$ are interchanged. However the unnatural character of these perturbations motivated us not to include their treatment in this paper.

In order to obtain a limiting absorption principle for $H$, one has to invoke some abstract results. An optimal regularity condition of $H$ with respect to $A$ has to be satisfied. We refer to [1 Chap. 5] for the definitions of $\mathscr{C}^{1,1}(A)$ and $\mathscr{C}^{1,1}\left(A ; \mathcal{D}\left(H_{0}\right), \mathcal{D}\left(H_{0}\right)^{*}\right)$, and for more explanations on regularity conditions.

Proposition 3.3. Let $V$ be a short-range or a long-range potential. Then $H$ is of class $\mathscr{C}^{1,1}(A)$.

Proof. Since $\left\{e^{i t A}\right\}_{t \in \mathbb{R}}$ leaves $\mathcal{D}(H)=\mathcal{D}\left(H_{0}\right)$ invariant, it is equivalent to prove that $H$ belongs to $\mathscr{C}^{1,1}\left(A ; \mathcal{D}\left(H_{0}\right), \mathcal{D}\left(H_{0}\right)^{*}\right)$ [1, Thm. 6.3.4.(b)]. But in Proposition [2.5(c), it has already been shown that $H_{0}$ is of class $C^{2}(A)$, so that $H_{0}$ is of class $\mathscr{C}^{1,1}\left(A ; \mathcal{D}\left(H_{0}\right), \mathcal{D}\left(H_{0}\right)^{*}\right)$. Thus it is enough to prove that $V$ belongs to $\mathscr{C}^{1,1}\left(A ; \mathcal{D}\left(H_{0}\right), \mathcal{D}\left(H_{0}\right)^{*}\right)$. In the short-range case, we shall use [1, Thm. 7.5.8], which implies that $V$ belongs to $\mathscr{C}^{1,1}\left(A ; \mathcal{D}\left(H_{0}\right), \mathcal{D}\left(H_{0}\right)^{*}\right)$. The conditions needed for that theorem are obtained in points (i) and (ii) below. In the long-range case, the claim follows by [1, Thm. 7.5.7], which can be applied because of points (i), (iii), (iv) and (v) below.

(i) We first check that $\left\{e^{i t\left\langle Q_{3}\right\rangle}\right\}_{t \in \mathbb{R}}$ is a polynomially bounded $C_{0}$-group in $\mathcal{D}\left(H_{0}\right)$ and in $\mathcal{D}\left(H_{0}\right)^{*}$. Lemma 2.3 (a) (with $n=0$ and $f\left(Q_{3}\right)=\left\langle Q_{3}\right\rangle$ ) implies that $H_{0}$ is of class $C^{1}\left(\left\langle Q_{3}\right\rangle\right)$. Furthermore, by an argument similar to that given in part (b) of the proof of Proposition 2.5. one shows that $\left\{e^{i t\left\langle Q_{3}\right\rangle}\right\}_{t \in \mathbb{R}}$ leaves $\mathcal{D}\left(H_{0}\right)$ invariant. Since $H_{0} e^{i t\left\langle Q_{3}\right\rangle}-e^{i t\left\langle Q_{3}\right\rangle} H_{0}$, defined on $\mathscr{D}$, extends continuously to the operator $t \alpha_{3} Q_{3}\left\langle Q_{3}\right\rangle^{-1} e^{i t\left\langle Q_{3}\right\rangle} \in \mathscr{B}(\mathcal{H})$, one gets that $\left\|e^{i t\left\langle Q_{3}\right\rangle}\right\|_{\mathscr{B}\left(\mathcal{D}\left(H_{0}\right)\right)} \leq$ Const. $\langle t\rangle$ for all $t \in \mathbb{R}$, 
i.e. the polynomial bound of the $C_{0}$-group in $\mathcal{D}\left(H_{0}\right)$. By duality, $\left\{e^{i t\left\langle Q_{3}\right\rangle}\right\}_{t \in \mathbb{R}}$ extends to a polynomially bounded $C_{0}$-group in $\mathcal{D}\left(H_{0}\right)^{*}$ [1] Prop. 6.3.1]. The generators of these $C_{0}$-groups are densely defined and closed in $\mathcal{D}\left(H_{0}\right)$ and in $\mathcal{D}\left(H_{0}\right)^{*}$ respectively; both are simply denoted by $\left\langle Q_{3}\right\rangle$.

(ii) Since $\left\{e^{i t A}\right\}_{t \in \mathbb{R}}$ leaves $\mathcal{D}\left(H_{0}\right)$ invariant, one may also consider the $C_{0}$-group in $\mathcal{D}\left(H_{0}\right)$ obtained by restriction and the $C_{0}$-group in $\mathcal{D}\left(H_{0}\right)^{*}$ obtained by extension. The generator of each of these $C_{0}$-groups will be denoted by $A$. Let $\mathcal{D}\left(A ; \mathcal{D}\left(H_{0}\right)\right):=\left\{\varphi \in \mathcal{D}\left(H_{0}\right) \cap \mathcal{D}(A): A \varphi \in \mathcal{D}\left(H_{0}\right)\right\}$ be the domain of $A$ in $\mathcal{D}\left(H_{0}\right)$, and let $\mathcal{D}\left(A^{2} ; \mathcal{D}\left(H_{0}\right)\right):=\left\{\varphi \in \mathcal{D}\left(H_{0}\right) \cap \mathcal{D}\left(A^{2}\right): A \varphi, A^{2} \varphi \in \mathcal{D}\left(H_{0}\right)\right\}$ be the domain of $A^{2}$ in $\mathcal{D}\left(H_{0}\right)$. We now check that $\left\langle Q_{3}\right\rangle^{-1} A$ and $\left\langle Q_{3}\right\rangle^{-2} A^{2}$, defined on $\mathcal{D}\left(A ; \mathcal{D}\left(H_{0}\right)\right)$ and on $\mathcal{D}\left(A^{2} ; \mathcal{D}\left(H_{0}\right)\right)$ respectively, extend to operators in $\mathscr{B}\left(\mathcal{D}\left(H_{0}\right)\right)$. After some commutator calculations performed on $\mathscr{D}$ and involving Lemma 2.3 one first obtains that $\left\langle Q_{3}\right\rangle^{-1} A$ and $\left\langle Q_{3}\right\rangle^{-2} A$ are respectively equal on $\mathscr{D}$ to some operators $S_{1}$ and $S_{2}\left\langle Q_{3}\right\rangle^{-1}$ in $\mathscr{B}(\mathcal{H})$, where $S_{1}$ and $S_{2}$ are polynomials in $H_{0}^{-1}, P_{3} H_{0}^{-1}, \alpha_{3}$ and $f\left(Q_{3}\right)$ for bounded functions $f$ with bounded derivatives. Since $\mathscr{D}$ is a core for $A$, these equalities even hold on $\mathcal{D}(A)$. Hence one has on $\mathcal{D}\left(A^{2}\right)$ :

$$
\left\langle Q_{3}\right\rangle^{-2} A^{2}=\left(\left\langle Q_{3}\right\rangle^{-2} A\right) A=S_{2}\left\langle Q_{3}\right\rangle^{-1} A=S_{2} S_{1} .
$$

In consequence, $\left\langle Q_{3}\right\rangle^{-1} A$ and $\left\langle Q_{3}\right\rangle^{-2} A^{2}$ are equal on $\mathcal{D}(A)$ and on $\mathcal{D}\left(A^{2}\right)$ respectively, to operators expressed only in terms of $H_{0}^{-1}, P_{3} H_{0}^{-1}, \alpha_{3}$ and $f\left(Q_{3}\right)$ for bounded functions $f$ with bounded derivatives. Moreover, one easily observes that these operators and their products belong to $\mathscr{B}\left(\mathcal{D}\left(H_{0}\right)\right)$. Thus, it follows that $\left\langle Q_{3}\right\rangle^{-1} A$ and $\left\langle Q_{3}\right\rangle^{-2} A^{2}$ are equal on $\mathcal{D}\left(A ; \mathcal{D}\left(H_{0}\right)\right)$ and on $\mathcal{D}\left(A^{2} ; \mathcal{D}\left(H_{0}\right)\right)$ respectively to some operators belonging to $\mathscr{B}\left(\mathcal{D}\left(H_{0}\right)\right)$.

(iii) By duality, the operator $\left(\left\langle Q_{3}\right\rangle^{-1} A\right)^{*}$ belongs to $\mathscr{B}\left(\mathcal{D}\left(H_{0}\right)^{*}\right)$. Now, for $\psi \in \mathcal{D}\left(H_{0}\right)^{*}$ and $\varphi \in \mathcal{D}\left(A ; \mathcal{D}\left(H_{0}\right)\right)$, one has

$$
\left\langle\left(\left\langle Q_{3}\right\rangle^{-1} A\right)^{*} \psi, \varphi\right\rangle=\left\langle\psi,\left\langle Q_{3}\right\rangle^{-1} A \varphi\right\rangle=\left\langle\left\langle Q_{3}\right\rangle^{-1} \psi, A \varphi\right\rangle,
$$

where $\langle\cdot, \cdot\rangle$ denotes the duality between $\mathcal{D}\left(H_{0}\right)$ and $\mathcal{D}\left(H_{0}\right)^{*}$. Since $\left\langle Q_{3}\right\rangle^{-1}$ is a homeomorphism from $\mathcal{D}\left(H_{0}\right)^{*}$ to the domain of $\left\langle Q_{3}\right\rangle$ in $\mathcal{D}\left(H_{0}\right)^{*}$, it follows from 3.13 that the domain of $\left\langle Q_{3}\right\rangle$ in $\mathcal{D}\left(H_{0}\right)^{*}$ is included in the domain of $A$ in $\mathcal{D}\left(H_{0}\right)^{*}$ (the adjoint of the operator $A$ in $\mathcal{D}\left(H_{0}\right)$ is equal to the operator $-A$ in $\left.\mathcal{D}\left(H_{0}\right)^{*}\right)$.

(iv) The inequality $r\left\|\left(\left\langle Q_{3}\right\rangle+i r\right)^{-1}\right\|_{\mathscr{B}\left(\mathcal{D}\left(H_{0}\right)^{*}\right)} \leq$ Const. for all $r>0$ is obtained from relation 3.15, given in the proof of Lemma2.3 with $f\left(Q_{3}\right)=\left(\left\langle Q_{3}\right\rangle+i r\right)^{-1}$.

(v) Assume that $V$ is a long-range (scalar) potential. Then the following equality holds in form sense on $\mathscr{D}$ :

$$
2[i V, A]=-Q_{3}\left(\partial_{3} V\right) H_{0}^{-1}-H_{0}^{-1} Q_{3}\left(\partial_{3} V\right)+\left[i V, H_{0}^{-1}\right] Q_{3} P_{3}+P_{3} Q_{3}\left[i V, H_{0}^{-1}\right],
$$

with $\left[i V, H_{0}^{-1}\right]=\sum_{j=1}^{3} H_{0}^{-1} \alpha_{j}\left(\partial_{j} V\right) H_{0}^{-1}$. Using Lemma2.3 (a), one gets that the last two terms in (3.14) are equal in form sense on $\mathscr{D}$ to

$$
2 \operatorname{Re} \sum_{j=1}^{3} H_{0}^{-1} \alpha_{j} Q_{3}\left(\partial_{j} V\right) P_{3} H_{0}^{-1}-2 \operatorname{Im} \sum_{j=1}^{3} H_{0}^{-1} \alpha_{j}\left(\partial_{j} V\right) H_{0}^{-1} \alpha_{3} P_{3} H_{0}^{-1} .
$$

It follows that $[i V, A]$, defined in form sense on $\mathscr{D}$, extends continuously to an operator in $\mathscr{B}(\mathcal{H})$. Now let $\vartheta$ be as in Definition 1.1 Then a direct calculation using the explicit form of $[i V, A]$ obtained above 
implies that

$$
\left\|\vartheta\left(\frac{\left\langle Q_{3}\right\rangle}{r}\right)[i V, A]\right\| \leq \mathrm{C} \sum_{j=1}^{3}\left\|\vartheta\left(\frac{\left\langle Q_{3}\right\rangle}{r}\right)\left\langle Q_{3}\right\rangle\left(\partial_{j} V\right)\right\|+\frac{\mathrm{D}}{r}
$$

for all $r>0$ and some positive constants $\mathrm{C}$ and D.

As a direct a consequence, one obtains that

Lemma 3.4. If $V$ satisfies the hypotheses of Theorem $\left\lfloor .2\right.$ then $A$ is conjugate to $H$ on $\mathbb{R} \backslash \sigma_{\mathrm{sym}}^{0}$.

Proof. Proposition 3.3 implies that both $H_{0}$ and $H$ are of class $\mathscr{C}^{1,1}(A)$. Furthermore, the difference $(H+i)^{-1}-\left(H_{0}+i\right)^{-1}$ is compact by Proposition 3.1 and $\varrho_{H_{0}}^{T}>0$ on $\mathbb{R} \backslash \sigma_{\text {sym }}^{0}$ due to Proposition 2.7 Hence the claim follows by [1, Thm. 7.2.9\& Prop. 7.2.6].

We can finally give the proof of Theorem 1.2

Proof of Theorem 1.2 Since $A$ is conjugate to $H$ on $\mathbb{R} \backslash \sigma_{\mathrm{sym}}^{0}$ by Lemma3.4 the assertions (a) and (b) follow by the abstract conjugate operator method [1] Cor. 7.2.11 \& Thm. 7.4.2].

The limiting absorption principle directly obtained via [1 Thm. 7.4.1] is expressed in terms of some interpolation space, associated with $\mathcal{D}(A)$, and of its adjoint. Since both are not standard spaces, one may use [1] Prop. 7.4.4] for the Friedrichs couple $\left(\mathcal{D}\left(\left\langle Q_{3}\right\rangle\right), \mathcal{H}\right)$ to get the statement (c). In order to verify the hypotheses of that proposition, one has to check that for each $z \in \mathbb{C} \backslash \sigma(H)$ the inclusion $(H-z)^{-1} \mathcal{D}\left(\left\langle Q_{3}\right\rangle\right) \subset \mathcal{D}(A)$ holds. However, since $\mathcal{D}\left(\left\langle Q_{3}\right\rangle\right)$ is included in $\mathcal{D}(A)$ by Proposition 2.4 it is sufficient to prove that for each $z \in \mathbb{C} \backslash \sigma(H)$ the operator $(H-z)^{-1}$ leaves $\mathcal{D}\left(\left\langle Q_{3}\right\rangle\right)$ invariant. Since $\mathcal{D}(H)=\mathcal{D}\left(H_{0}\right)$ is left dinvariant by the group $\left\{e^{i t\left\langle Q_{3}\right\rangle}\right\}_{t \in \mathbb{R}}$ (see Proposition 3.3(i)) one easily gets from [1, Thm. 6.3.4.(a)] that $H$ is of class $C^{1}\left(\left\langle Q_{3}\right\rangle\right)$, which implies the required invariance of $\mathcal{D}\left(\left\langle Q_{3}\right\rangle\right)$ [1 Thm. 6.2.10.(b)].

\section{Appendix}

Proof of Lemma 2.2 (a) Let $\varphi, \psi$ be in $\mathscr{D}$. Using the transformation 2.1 , one gets

$$
\left\langle H_{0}^{-n} \varphi, Q_{3} \psi\right\rangle=\int_{\mathbb{R}}\left\langle H_{0}(\xi)^{-n}(\mathscr{F} \varphi)(\xi),\left(i \partial_{\xi} \mathscr{F} \psi\right)(\xi)\right\rangle_{\mathcal{H}_{12}} \mathrm{~d} \xi .
$$

Now the map $\mathbb{R} \ni \xi \mapsto H_{0}(\xi)^{-n} \in \mathscr{B}\left(\mathcal{H}_{12}\right)$ is norm differentiable with its derivative equal to $-\sum_{j=1}^{n} H_{0}(\xi)^{-j} \alpha_{3} H_{0}(\xi)^{j-n-1}$. Hence $\left\{\partial_{\xi}\left[H_{0}(\xi)^{-n}(\mathscr{F} \varphi)(\xi)\right]\right\}_{\xi \in \mathbb{R}}$ belongs to $\int_{\mathbb{R}}^{\oplus} \mathcal{H}_{12} \mathrm{~d} \xi$. Thus one can perform an integration by parts (with vanishing boundary contributions) and obtain

$$
\left\langle H_{0}^{-n} \varphi, Q_{3} \psi\right\rangle=\int_{\mathbb{R}}\left\langle i \partial_{\xi}\left[H_{0}(\xi)^{-n}(\mathscr{F} \varphi)(\xi)\right],(\mathscr{F} \psi)(\xi)\right\rangle_{\mathcal{H}_{12}} \mathrm{~d} \xi
$$

It follows that $\left|\left\langle H_{0}^{-n} \varphi, Q_{3} \psi\right\rangle\right| \leq$ Const. $\|\psi\|$ for all $\psi \in \mathscr{D}$. Since $Q_{3}$ is essentially self-adjoint on $\mathscr{D}$, this implies that $H_{0}^{-n} \varphi$ belongs to $\mathcal{D}\left(Q_{3}\right)$. 
(b) The boundedness of $P_{3} H_{0}^{-1}$ is a consequence of the estimate

$$
\underset{\xi \in \mathbb{R}}{\operatorname{essip}}\left\|\xi H_{0}(\xi)^{-1}\right\|_{\mathscr{B}\left(\mathcal{H}_{12}\right)}=\operatorname{ess} \sup _{\xi \in \mathbb{R}}\left\|\frac{|\xi|}{\left[H_{0}(0)^{2}+\xi^{2}\right]^{1 / 2}}\right\|_{\mathscr{B}\left(\mathcal{H}_{12}\right)}<\infty
$$

and of the direct integral formalism [6 Prop. $3.6 \& 3.7]$. The remaining assertions follow by standard arguments.

Proof of Lemma 2.3 (a) One first observes that the following equality holds on $\mathscr{D}$ :

$$
i H_{0}^{-1} f\left(Q_{3}\right) H_{0}=-H_{0}^{-1} \alpha_{3} f^{\prime}\left(Q_{3}\right)+i f\left(Q_{3}\right)
$$

Now, for $\varphi, \psi \in \mathscr{D}$ and $\eta \in H_{0}^{-n} \mathscr{D}$, one has

$$
\begin{aligned}
& \left\langle\varphi, i H_{0}^{-1} f\left(Q_{3}\right) \eta\right\rangle-\left\langle\varphi, i f\left(Q_{3}\right) H_{0}^{-1} \eta\right\rangle \\
& =\left\langle\varphi, i H_{0}^{-1} f\left(Q_{3}\right) H_{0} \psi\right\rangle+\left\langle\varphi, i H_{0}^{-1} f\left(Q_{3}\right)\left(\eta-H_{0} \psi\right)\right\rangle-\left\langle\bar{f}\left(Q_{3}\right) \varphi, i H_{0}^{-1} \eta\right\rangle \\
& =-\left\langle\varphi, H_{0}^{-1} \alpha_{3} f^{\prime}\left(Q_{3}\right) H_{0}^{-1} \eta\right\rangle-\left\langle\varphi, H_{0}^{-1} \alpha_{3} f^{\prime}\left(Q_{3}\right) H_{0}^{-1}\left(H_{0} \psi-\eta\right)\right\rangle \\
& \quad+\left\langle\bar{f}\left(Q_{3}\right) \varphi, i H_{0}^{-1}\left(H_{0} \psi-\eta\right)\right\rangle+\left\langle\bar{f}\left(Q_{3}\right) H_{0}^{-1} \varphi, i\left(\eta-H_{0} \psi\right)\right\rangle,
\end{aligned}
$$

where we have used $(3.15)$ in the last equality for the term $\left\langle\varphi, i H_{0}^{-1} f\left(Q_{3}\right) H_{0} \psi\right\rangle$. Hence there exists a constant $\mathrm{C}$ (depending on $\varphi$ ) such that

$$
\left|\left\langle\varphi, i H_{0}^{-1} f\left(Q_{3}\right) \eta\right\rangle-\left\langle\varphi, i f\left(Q_{3}\right) H_{0}^{-1} \eta\right\rangle+\left\langle\varphi, H_{0}^{-1} \alpha_{3} f^{\prime}\left(Q_{3}\right) H_{0}^{-1} \eta\right\rangle\right| \leq \mathrm{C}\left\|\eta-H_{0} \psi\right\| .
$$

Then the statement is a direct consequence of the density of $H_{0} \mathscr{D}$ and $\mathscr{D}$ in $\mathcal{H}$.

(b) This is a simple corollary of the point (a).

\section{Acknowledgements}

We thank M. Măntoiu for having suggested to us the present study. We are also grateful to W. Amrein, H. D. Cornean and B. Thaller for their helpful remarks. This work was partially supported by the Swiss National Science Foundation.

\section{References}

[1] W.O. Amrein, A. Boutet de Monvel and V. Georgescu, $C_{0}$-groups, commutator methods and spectral theory of N-body Hamiltonians, Progress in Math. vol. 135, Birkhäuser, 1996.

[2] M.S. Birman and T.A. Suslina, The periodic Dirac operator is absolutely continuous, Integr. Equ. Oper. Theory 34 (1999), 377-395.

[3] P. Briet and H.D. Cornean, Locating the spectrum for magnetic Schrödinger and Dirac operators, Comm. Partial Differential Equations 27 (2002), no. 5-6, 1079-1101. 
[4] A.M. Boutet de Monvel and R. Purice, A distinguished self-adjoint extension for the Dirac operator with strong local singularities and arbitrary behaviour at infinity, Rep. Math. Phys. 34 (1994), no. 3, 351-360.

[5] P.R. Chernoff, Schrödinger and Dirac operators with singular potentials and hyperbolic equations, Pacific J. Math. 72 (1977), no. 2, 361-382.

[6] T.R. Chow, A spectral theory for direct integrals of operators, Math. Ann. Phys. 188 (1970), 285303.

[7] L.I. Danilov, On the spectrum of the two-dimensional periodic Dirac operator, Theo. and Math. Physics 118 (1999), no. 1, 1-11.

[8] V. Georgescu and C. Gérard, On the Virial theorem in quantum mechanics, Commun. Math. Phys. 208 (1999), 275-281.

[9] V. Georgescu and M. Măntoiu, On the spectral theory of singular Dirac type Hamiltonians, J. Operator Theory 46 (2001), 289-321.

[10] A. Grigis and A. Mohamed, Finitude des lacunes dans le spectre de l'opérateur de Schrödinger et de celui de Dirac avec des potentiels électrique et magnétique périodiques, J. Math. Kyoto Univ. 33 (1993), no. 4, 1071-1096.

[11] B. Helffer, J. Nourrigat and X.P. Wang, Sur le spectre de l'équation de Dirac (dans $\mathbb{R}^{2}$ ou $\mathbb{R}^{3}$ ) avec champ magnétique, Ann. scient. Éc. Norm. Sup. 22 (1989), 515-533.

[12] M. Măntoiu and M. Pascu, Perturbations of magnetic Schrödinger operators, Lett. Math. Phys. 54 (2000), 181-192.

[13] E. Mourre, Absence of singular continuous spectrum for certain self-adjoint operators, Commun. Math. Phys. 78 (1981), 391-408.

[14] M. Reed and B. Simon, Methods of modern mathematical physics, II \& IV, Academic Press, New York, 1972-1978.

[15] B. Thaller, The Dirac Equation, Springer-Verlag, 1992.

[16] K. Yokoyama, Limiting absorption principle for Dirac operator with constant magnetic field and long-range potential, Osaka J. Math. 38 (2001), 649-666. 